\title{
Enduring Value of Thiopurines for Inflammatory Bowel Disease Therapy
}

\author{
Burton I. Korelitz ${ }^{1}$
}

Published online: 26 December 2016

(c) Springer Science+Business Media New York 2016

Prior to the advent of the use of infliximab and other biologics for the treatment of ulcerative colitis, the immunosuppressive drugs, azathioprine (AZA) and 6-mercaptopurine (6-MP), provided long-term remissions beyond that accomplished by sulfasalazine, 5-aminosalicylates, and corticosteroids [1]. The response to these two thiopurines was neither marked nor rapid, but once accomplished was enduring. Since the efficacy of therapeutic antibodies (biologics) has been well substantiated, the rate of response faster, and the adverse reactions minimal, it was logical that they be favored for the initial therapy. What then should be the remaining utility of AZA and 6-MP?

As experience accumulated with biologics, it was recognized that not all patients responded, and furthermore, in many there was a loss of response following initial improvement. Furthermore, in some cases, the use of biologics was complicated by allergic and other adverse reactions, providing further opportunities for the use of the thiopurines alone, for 6-MP or AZA in conjunction with the biologics-shown to be more effective than either drug alone [2]. Thiopurine cotherapy also reduces allergic reactions to the biologics associated with elevated serum concentrations of antibodies facilitating drug excretion with consequent reduction of the drug serum concentrations [3].

In the current issue of Digestive Diseases and Sciences, Prieux-Klotz et al. [4] provide new evidence that thiopurines by themselves retain importance in the maintenance

Burton I. Korelitz

bkorelitz@northwell.edu

1 Division of Gastroenterology, Department of Medicine, Lenox Hill Hospital, New York, NY 10075, USA therapy of ulcerative colitis, associated with mucosal healing in $43.7 \%$ and histological healing in $38 \%$. In this study, the thiopurine dose was determined by the patient's weight, as was established in the original trial of 6-MP for Crohn's disease (CD) [1], although later experience revealed that further dose increases further augmented the clinical response, including mucosal healing. In the 6-MP trial for $\mathrm{CD}$, we initially used a standardized dose of $1.5 \mathrm{mg} / \mathrm{kg}$, modified with subsequent experience to dose modification based on the balance between response on one hand and leukopenia on the other $[5,6]$. The use of standardized and weight-based dosing of 6-MP and AZA for the purpose of trials has compromised the potential value of immunosuppressives [5], since in real-world management of both ulcerative colitis (UC) and CD, dose augmentation has clearly improved the clinical response and induced more remissions. No studies as yet have been undertaken whereby the dose is adjusted as needed according to a protocol.

Furthermore, in the study by Prieux-Klotz et al. [4], only 2 of the 80 patients were treated with 6-MP, while the remaining 78 received AZA. Differences between AZA and 6-MP never were resolved. Though we know that AZA is metabolized to 6-MP in a $\sim 2: 1$ ratio, the relative amounts and therapeutic efficacy of the metabolites has not been thoroughly investigated. In my experience, the performance of 6-MP after longstanding maintenance has been superior to that of AZA when our results have been compared with others who used only AZA [6, 7].

I have long witnessed the measurement of response to treatment as documented by clinical improvement, physicians' global evaluation including laboratory tests and imaging, and to the endoscopic mucosal healing as utilized in this trial. Most researchers in inflammatory bowel disease now recognize the value of histological healing as 
well as endoscopic mucosal healing in assessing full response and remission [8, 9]. Histologic healing was accomplished in $38 \%$ of the patients studied [4], which represents a minimum number given that only $47 \%$ of subjects were assessed for this outcome.

Despite the lack of resolution of these issues for the purpose of this trial of thiopurines (fixed dose, 6-MP versus AZA, and lack of full access to all of the data with regard to histological healing), this study does provide renewed evidence for an unceasing benefit of thiopurines in the treatment of ulcerative colitis, whether alone or to supplement the response to biologicals should the clinical circumstances warrant [4, 7].

\section{References}

1. Present DH, Korelitz BI, Wisch N, et al. Treatment of Crohn's disease with 6-mercaptopurine. N Engl J Med. 1980;302:981-987.

2. Colombel JF, Sandborn WJ, Reinisch W, et al. Infliximab, azathioprine, or combination therapy for Crohn's disease. $N$ Engl J Med. 2010;362:1383-1395.
3. Ben-Horin S, Wagerman M, Kopylov U, et al. Addition of an immune-modulator to infliximab therapy eliminates antidrug antibodies in serum and restores clinical response of patients with inflammatory bowel disease. Clin Gastroenterol Hepatol. 2013;11:444-447.

4. Prieux-Klotz C, Nahon S, Amiot A, et al. Rate and predictors of mucosal healing in ulcerative colitis treated with thiopurines: results of a multicentric cohort study. Dig Dis Sci. (Epub ahead of print). doi:10.1007/s10620-016-4374-0.

5. Adler DJ, Korelitz BI. The therapeutic efficacy of 6-mercaptopurine in refractory ulcerative colitis. Am J Gastroenterol. 1990;85:717-722.

6. Korelitz BI. Expert opinion-experience with 6-mercaptopurine in the treatment of inflammatory bowel disease. World J Gastroenterol. 2013;19:2979-2984.

7. Korelitz BI, Present DH. 6-Mercaptopurine/azathioprine remains an important contributor in managing Crohn's disease. J Crohn's Colitis. 2014;8:735-738.

8. Korelitz BI. Mucosal healing as an index of colitis activity: back to histological healing for future indices. Inflamm Bowel Dis. 2010;16:1628-1630.

9. Bryant RV, Burger DC, Delo J, et al. Beyond endoscopic mucosal healing in UC: histological remission better predicts corticosteroid use and hospitalization over 6 years of follow-up. Gut. 2016;65:408-414. 